\title{
Ultrapure dialysis water obtained with additional ultrafilter may reduce inflammation in patients on hemodialysis
}

\author{
Biagio Di Iorio $^{1} \cdot$ Lucia Di Micco $^{1} \cdot$ Dario Bruzzese $^{2} \cdot$ Luca Nardone $^{1} \cdot$ Luigi Russo $^{3}$. \\ Pietro Formisano $^{4} \cdot$ Vittoria D'Esposito $^{4} \cdot$ Domenico Russo $^{3}[$
}

Received: 17 April 2017 / Accepted: 11 July 2017 / Published online: 23 August 2017

(C) The Author(s) 2017. This article is an open access publication

\begin{abstract}
Background Patients on standard dialysis, in particular those on high-flux and high-efficiency dialysis, are exposed to hundreds of liters of dialysis-water per week. The quality of dialysis-water is a factor responsible for inflammation in dialysis patients. Inflammation is a potent trigger of atherosclerosis and a pathogenetic factor in anemia, increasing mortality and morbidity in dialysis patients. Current systems for water treatment do not completely eliminate bacteria and endotoxins. This prospective study tested whether improved dialysis-water purity by an additional ultrafilter can reduce inflammation and ameliorate hemoglobin levels, with a consequent reduction in erythropoietin-stimulating agents (ESA).

Methods An ultrafilter, composed of two serially positioned devices with polysulfone membranes of 2.0 and $1.0 \mathrm{~m}^{2}$, respectively, was positioned within the fluid pathway before the dialysis machine. Prevalent dialysis patients were assigned either to continue dialysis with conventional dialysis-water (control phase) or to initiate dialysis sessions
\end{abstract}

Electronic supplementary material The online version of this article (doi:10.1007/s40620-017-0422-x) contains supplementary material, which is available to authorized users.

Domenico Russo

domenicorusso51@hotmail.com

1 Department of Nephrology, “A. Landolfi” Hospital (Solofra, Avellino), Solofra, Italy

2 Department of Statistics, University of Naples "FEDERICO II", Naples, Italy

3 Department of Public Health, University of Naples "FEDERICO II", Naples, Italy

4 Department of Genetics, University of Naples "FEDERICO II", Naples, Italy with improved dialysis-water purity (study phase). After 6 months, patients were crossed over. Total study duration was 1 year. Routine chemistry, bacterial count, endotoxin levels in dialysis-water as well as blood levels of pro- and antiinflammatory cytokines, human serum amyloid A, C-reactive protein and fraction 5 of complement were measured.

Results Thirty-two patients completed the study. Mean bacterial count was lower and endotoxin levels were absent in dialysis-water obtained with the ultrafilter. At the end of the study-phase, C-reactive protein and pro-inflammatory cytokines decreased while anti-inflammatory ones increased. Hemoglobin levels were improved with lower ESA doses.

Conclusions An additional ultrafilter improved dialysiswater purity, reduced levels of inflammation markers, ameliorated hemoglobin concentration with reduced ESA doses. These results remain speculative but they may generate studies to assess whether improved dialysis-water quality with an ultrafilter can reduce inflammation and improve survival of dialysis patients.

Keywords Ultrafilter · Dialysis water · Ultrapure water · Dialysis patients · Inflammation · Cytokines · Erythropoietin stimulating agents

\section{Introduction}

Inflammation plays a critical role in mortality and morbidity of dialysis patients, being a potent trigger of atherosclerosis and a pathogenetic factor in anemia [1-5]. There are several factors linked to uremia that can cause inflammation; some can be regarded as modifiable risk factors. This is the case of quality of dialysis-water. Current systems for water treatment do not completely remove bacteria and endotoxins [6-8]. Therefore, the quality of dialysis-water may be 
a significant factor responsible for inflammation. Indeed, patients on conventional dialysis, in particular those on high-flux and high-efficiency dialysis, are exposed to hundreds of liters of dialysis-water per week. Therefore, every effort should be made by clinicians to improve the purity of dialysis-water in order to minimize the negative effects of inflammation.

This study aimed to test in patients on dialysis whether an additional ultrafilter can decrease blood levels of markers of inflammation, increase hemoglobin levels $(\mathrm{Hb})$ and reduce doses of erythropoietin-stimulating agents (ESA).

\section{Methods}

This was a prospective, crossover study in prevalent dialysis patients. Patients signed written informed consent. The local ethic committee approved the study. Exclusion criteria were: inflammatory diseases; therapy with anti-inflammatory drugs or steroids; signs of malnutrition such as abnormal serum levels of albumin, cholesterol or triglycerides and change in dialysis prescription or ESA therapy within the 3 months preceding the study.

To improve the dialysis-water quality, an ultrafilter (Estorclean PLUS) was positioned within the fluid pathway before the dialysis machine. The ultrafilter was composed of two serially positioned devices with polysulfone membranes [MediSulfone, Medica, Medolla (MO), Italy] of 2.0 and $1.0 \mathrm{~m}^{2}$, respectively (Fig. 1). Characteristics of the ultrafilter were: bacteria retention capacity $>1010$
$\mathrm{CFU} / \mathrm{ml}$ (Brevundimonas diminuta), viruses retention capacity $>108$ (PhiX-174), endotoxin retention capacity $>105 \mathrm{EU} / \mathrm{ml}$. The ultrafilter remained in situ for the duration of the study.

Each patient was assigned either to dialysis sessions with conventional dialysis-water (control phase) or to dialysis sessions with improved dialysis-water quality (study phase). After a 6-month observation period, each patient was switched to dialysis sessions with the other dialysiswater. The total study duration was 1 year. Routine chemistry, pro- and anti-inflammatory cytokines, tumor necrosis factor (TNF)- $\alpha$, serum A-amyloid (SAA), and complement fraction 5 (C5a) were measured at the beginning and end of each study phase; blood samples were collected at the mid-week dialysis session. Mean duration of the dialysis sessions was $4 \mathrm{~h}, 3$ times a week. The dialysis membranes were: polyphenylene ( $n=14$ patients), polycarbonate $(n=6)$, polymethylmethacrylate $(n=6)$, and polyamide $(n=6)$. Changes in dialysis membrane were not permitted during the study.

Dialysis-water was obtained by reverse osmosis. Water quality was checked bi-monthly by an independent laboratory (Department of Public Health, University of Naples "Federico II"). Total microbial count was measured, and the limulus amebocyte lysate test (LAL-test; Pyrotell ${ }^{\circledR}$ Multitest Vial; Cape Cod Inc., Falmouth, MA, USA) was performed on samples collected from water entering into the dialysis machine. Three determinations were performed during both the control and study phase. A combination of chemical and heat disinfection of the dialysis machine was performed at the end of each patient treatment. A special disinfection

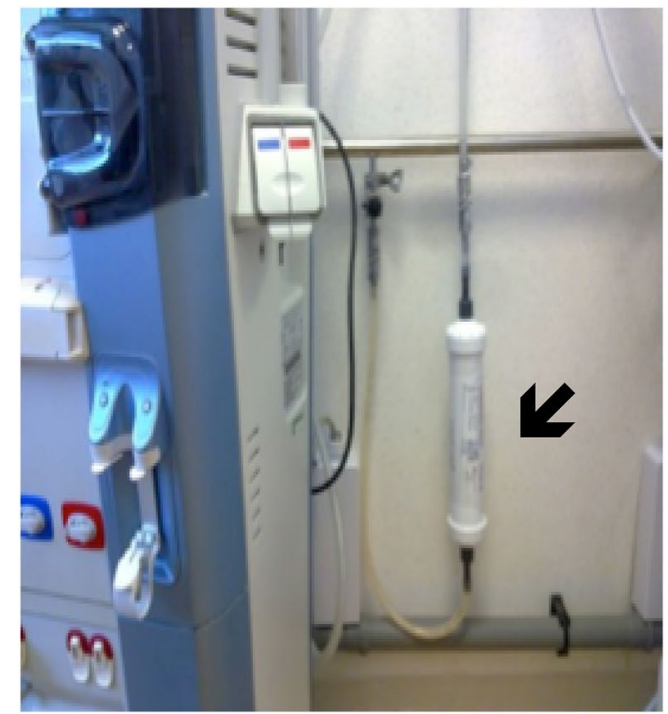

Fig. 1 Composition and position of ultrafilter. The ultrafilter was composed of two serially positioned devices with polysulfone membranes [MediSulfone, Medica, Medolla (MO), Italy] of 2.0 and $1.0 \mathrm{~m}^{2}$, respectively. Characteristics of the ultrafilter were: bacteria

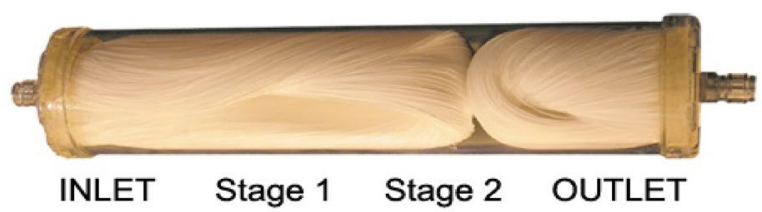

retention capacity $>1010 \mathrm{CFU} / \mathrm{ml}$ (Brevundimonas diminuta), viruses retention capacity $>108$ ( $\mathrm{PhiX} 174$ ), endotoxin retention capacity $>105 \mathrm{EU} / \mathrm{ml}$ 
cycle (lasting $45 \mathrm{~min}$ ) was performed twice a month in each machine with heat and chemical disinfection.

For cytokines and growth factors (Bio-Rad, Hercules, CA, USA) serum samples were analyzed in duplicate and diluted 4x; for SAA and C5a (HyCult Biotech, Uden, Netherlands) samples were analyzed in duplicate and diluted $40 \times$ and $100 \times$, respectively. Dilutions were performed with the specific dilution buffer provided by the manufacturers.

\section{Statistical analysis}

Numerical variables are summarized using median; categorical variables are described using absolute frequencies and percentages. In order to properly account for the crossover study-design and skewed distribution of variables, the non-parametric approach was used. In particular, the presence of a difference in carry-over effect was first evaluated using the Wilcoxon rank-sum test on the subject totals (summing up the two periods) while the significance of treatment effect was assessed with the Wilcoxon ranksum test on the subjects' period differences. Corresponding point estimates and confidence intervals (CI) were computed using the Hodges and Lehman estimator. Finally, non parametric analysis of the cross-over difference allowed us to assess the presence of a period effect. The decision to use a non parametric approach for the analysis of the cross-over design was related to the markedly skewed distribution of the outcome variables. However, the inferential properties of non parametric tests remain the same as the parametric counterparts; the difference concerns only the objective of the inference which focuses on the average in the case of paired and unpaired t test, and looks for a general location shift of the variable's distribution in the case of the Wilcoxon procedure.

As a sensitivity analysis, we also applied a linear mixed model on log-transformed values of variables but the results were similar and thus only the former have been reported. All tests were two sided and statistical significance was set at $\mathrm{p}<0.05$. Statistical analysis was performed using $\mathrm{R}$ statistical computing software (R Foundation for Statistical Computing, Vienna, Austria).

\section{Results}

From a cohort of 48 prevalent patients on dialysis in a single nephrology unit, 16 patients were excluded because of recent central venous catheter infection $(n=12)$ and pneumonia $(n=4)$. Thirty-two patients (mean age was $72 \pm 15$ years; $M=12$ ) entered the final evaluation. Dialysis vintage was $48 \pm 32$ months. During the study, vascular access remained patent, no patient was hospitalized or transplanted, and no death was registered. Mean value of $\mathrm{Kt} / \mathrm{V}$ (measured every two months) was $1.37 \pm 0.3$ and $1.38 \pm 0.5$ during the control and study phases, respectively. At the end of the study phase, the level of C-reactive protein (CRP) was lower than at the end of the control phase [0.89 (0.8-1.1) vs. $2.5(1.0-6.5)$ $\mathrm{mg} / \mathrm{l}, \mathrm{p}<0.01]$. Characteristics of conventional water and water obtained with the ultrafilter are presented in Table 1. Baseline and final routine chemistry and cytokine levels are reported in Tables 2 and 3, respectively. Characteristics of the membrane did not affect the cytokine levels (Table A, supplemental material).

All patients were treated with ESA: 19 with epoietinalpha (8 with biosimilars, 11 with brand); 10 with darboepoietin alpha; and 3 with methoxy polyethylene glycolepoetin beta. Changes in $\mathrm{Hb}$ concentration, ESA doses and ESA resistance index are reported in Table 4. At the end of the study phase, $\mathrm{Hb}$ significantly increased with lower ESA doses and ESA resistance index. The monthly trends in $\mathrm{Hb}$ concentration, ESA doses and ESA resistance index are reported in Table B (supplemental material).

\section{Discussion}

Current water treatment systems provide high quality dialysis-water. Nonetheless, bacterial products or short bacterial DNA fragments cannot be completely eliminated. Due to
Table 1 Characteristics of conventional water and water obtained with ultrafilter

\begin{tabular}{llll}
\hline & $\begin{array}{l}\text { Water without addi- } \\
\text { tional ultrafilter }\end{array}$ & $\begin{array}{l}\text { Water with additional } \\
\text { ultrafilter }\end{array}$ & Reference range \\
\hline Total microbial counts (CFU/ml) & 22 & 20 & $100 / \mathrm{ml}$ \\
Escherichia coli & 0 & 0 & $0 / 100 \mathrm{ml}$ \\
Staphylococcus aureus & 0 & 0 & $0 / 100 \mathrm{ml}$ \\
Pseudomonas Aeruginosa & 0 & 0 & $0 / 100 \mathrm{ml}$ \\
Fungi (mycetes) & 0 & 0 & $0 / 100 \mathrm{ml}$ \\
Endotoxin levels (EU/ml) (Limulus & 0.125 & 0 & $<0.25 \mathrm{EU} / \mathrm{ml}$ \\
amebocyte assay; LAL test) & & & \\
\hline
\end{tabular}

Values are the mean of measurements performed every 2 months both during the control phase and study phase 
Table 2 Biochemistry in control and study phase

\begin{tabular}{|c|c|c|c|c|c|c|}
\hline & Control phase & Study phase & Treatment effect $(95 \% \mathrm{CI})$ & $\mathrm{p}$ value & $\begin{array}{l}\text { Carry-over } \\
\text { p value }\end{array}$ & $\begin{array}{l}\text { Period effect } \\
\mathrm{p} \text { value }\end{array}$ \\
\hline Serum creatinine $(\mathrm{mg} / \mathrm{dl})$ & $8.3(7.2 ; 9.5)$ & $8.4(7 ; 9.2)$ & $0(-0.15$ to 0.15$)$ & 0.895 & 0.734 & 0.126 \\
\hline Serum albumin $(\mathrm{g} / \mathrm{dl})$ & $3.8(3.6 ; 4.0)$ & $4.0(3.8 ; 4.2)$ & $0.2(0.08$ to 0.31$)$ & $<0.001$ & 0.486 & 1 \\
\hline Total cholesterol (mg/dl) & $155(112.5 ; 183.3)$ & $171.5(136.5 ; 198)$ & $21.32(-6.5$ to 45$)$ & 0.132 & 0.181 & 1 \\
\hline Triglycerides (mg/dl) & $213(147.8 ; 276.8)$ & $242.5(180 ; 290.5)$ & $21(19.5$ to 25$)$ & $<0.001$ & 0.611 & 0.153 \\
\hline Ferritin (ng/ml) & $289.5(220.8 ; 333)$ & $275(205.8 ; 299.5)$ & $-15(-48.5$ to -4$)$ & 0.003 & 0.865 & 0.94 \\
\hline Serum phosphorus (mg/dl) & $4.3(3.8 ; 5.3)$ & $4.4(3.5 ; 5.0)$ & $-0.10(-0.45$ to 0.33$)$ & 0.678 & 0.376 & 0.624 \\
\hline PTH $(\mathrm{pg} / \mathrm{ml})$ & $311.5(224.3 ; 424.3)$ & $349.5(241.8 ; 393.8)$ & 17.5 ( -73.5 to 116$)$ & 0.665 & 0.97 & 0.418 \\
\hline $\mathrm{Hb}(\mathrm{gr} / \mathrm{dl})$ & $11.3(10.9 ; 11.8)$ & $11.9(11.8 ; 12.1)$ & $0.65(0.50$ to 0.90$)$ & $<0.001$ & 0.299 & 0.179 \\
\hline
\end{tabular}

Numbers are median and $95 \% \mathrm{CI}$

$C I$ confidence interval, $P T H$ parathormone, $H b$ hemoglobin

Table 3 Cytokine levels in the control and study phase

\begin{tabular}{|c|c|c|c|c|c|c|}
\hline & Control phase & Study phase & Treatment effect $(95 \%$ CI) & $\mathrm{p}$ value & $\begin{array}{l}\text { Carry-over } \\
\text { p value }\end{array}$ & $\begin{array}{l}\text { Period effect } \\
\mathrm{p} \text { value }\end{array}$ \\
\hline \multicolumn{7}{|c|}{ Pro-inflammatory cytokines } \\
\hline IL-1 (pg/ml) & $9.7(8.9 ; 10.6)$ & $9.2(8.4 ; 9.8)$ & $-0.78(-1,12$ to -0.5$)$ & $<0.001$ & 0.809 & 0.651 \\
\hline IL-6 (pg/ml) & $43.7(36.7 ; 50.9)$ & $37.2(34.7 ; 44)$ & $-4.36(-7.09$ to -2.57$)$ & $<0.001$ & 0.305 & 0.258 \\
\hline IL-8 (pg/ml) & $60.9(49.4 ; 74.3)$ & $53.3(45 ; 70.5)$ & $-4.35(-6.1$ to -3.04$)$ & $<0.001$ & 0.184 & 0.642 \\
\hline IL-12 (pg/ml) & $102.1(74.3 ; 124.2)$ & $102.7(76.3 ; 125.4)$ & $-0.5(-1.65$ to 0.11$)$ & 0.076 & 0.21 & 0.91 \\
\hline MCP-1 (pg/ml) & $78.8(73.4 ; 83.1)$ & $76.3(71.6 ; 81.9)$ & $-1.84(-2.45$ to -1.4$)$ & $<0.001$ & 1 & 0.539 \\
\hline TNF-a (pg/ml) & $108.8(98.7 ; 128.8)$ & $97(93.8 ; 113.1)$ & $-7.26(-11.88$ to -4.08$)$ & $<0.001$ & 0.752 & 0.696 \\
\hline SAA (ng/ml) & $5949.1(3248.3 ; 10,000)$ & $2747.5(2089.2 ; 3771.9)$ & $-3491.2(-4438.5$ to -2132.4$)$ & $<0.001$ & 0.867 & 0.445 \\
\hline C5a (ng/ml) & $96.2(78.9 ; 121.4)$ & $60.3(48.3 ; 88.2)$ & $-29.27(-53.64$ to -18.87$)$ & $<0.001$ & 0.724 & 0.402 \\
\hline \multicolumn{7}{|c|}{ Anti-inflammatory cytokines } \\
\hline IL-1ra (pg/ml) & $251(222.2 ; 295)$ & $255.3(221.4 ; 296.4)$ & $5.12(-0.4$ to 10.62$)$ & 0.061 & 0.926 & 0.616 \\
\hline IL-4 (pg/ml) & $14.2(11 ; 17.5)$ & $16.2(13.2 ; 18.4)$ & $1.35(0.95$ to 1.75$)$ & $<0.001$ & 0.224 & 0.196 \\
\hline IL-10 (pg/ml) & $51.4(45.3 ; 61.4)$ & $53.3(50.1 ; 61.5)$ & $1.22(-2.9$ to 6.69$)$ & 0.402 & 0.254 & 0.468 \\
\hline IL-13 (pg/ml) & $18.5(15.7 ; 22.9)$ & $20(17.6 ; 25.6)$ & $1.28(0.69$ to 1.89$)$ & $<0.001$ & 0.128 & 0.956 \\
\hline IL-17 (pg/ml) & $178.6(140 ; 215.1)$ & $183.3(157.2 ; 222.8)$ & 3.57 (1.76 to 7.47$)$ & $<0.001$ & 0.376 & 0.491 \\
\hline
\end{tabular}

Numbers are median and $95 \%$ CI

$C I$ confidence interval, $I L$ interleukin, $M C P$ monocyte chemoattractant protein, $T N F$ tumor necrosis factor, $S A A$ serum A-amyloid, $C 5 a$ complement fraction 5

Table 4 Concentrations of hemoglobin, ESA doses and erythropoietin resistance index (ERI) at Control and Study phase

\begin{tabular}{lcrrl}
\hline Variable & Number & Control phase & Study phase & $\begin{array}{l}\text { Percent variation } \\
\text { study versus control } \\
\text { phase }\end{array}$ \\
\hline Hemoglobin (g/dl) & 32 & $11.2 \pm 0.9$ & $11.9 \pm 1.3^{\bullet}$ & +6.25 \\
Epoietin alpha (units per week) & 19 & $11,313 \pm 650$ & $8670 \pm 554^{\bullet}$ & -23.4 \\
Darboepoietin alpha (units per week) & 10 & $108 \pm 111$ & $85 \pm 69^{+}$ & -21.3 \\
$\begin{array}{l}\text { Methoxy polyethylene glycol-epoetin } \\
\text { beta (unit per month) }\end{array}$ & 3 & $233 \pm 130$ & $152 \pm 67$ & -34.8 \\
ERI: EPO (week/BW)/Hb & 29 & $13.1 \pm 7.5$ & $9.5 \pm 6.0^{\bullet}$ & \\
\hline
\end{tabular}

Numbers are mean \pm SD

$\bullet p<0.01$

${ }^{+} p<0.05$ 
their small size, bacterial products or short bacterial DNA fragments easily pass through the dialyzer membrane and into the bloodstream, amplifying the inflammation [6,9-11]. Several studies have evaluated and quantified the presence of bacterial products or short bacterial DNA fragments both in blood and dialysis-water adopting bacterial DNA primers for sequencing [6-10]; these expensive procedures have established that there is a strict correlation between contaminants and level of inflammation. In contrast, very few interventional studies have been carried out to assess the usefulness of additional devices able to produce ultrapure dialysis-water. In 25 patients on dialysis, ultrafilters reduced the levels of bacterial DNA fragments and endotoxins [11]. However, markers of inflammation were not measured in that study; nonetheless, the increased circulating endotoxin level was associated with systemic inflammation, that was regarded as responsible for vascular stiffness [11]. The latter is frequently observed in different stages of chronic kidney disease and more frequently has been found to be associated to altered mineral metabolism [12-14].

No study has evaluated, to our knowledge, the usefulness of preventive measures such as an additional ultrafilter in minimizing inflammation correlated to dialysis-water by measuring the changes of several circulating markers of inflammation at the same time. The present study evaluated the efficacy of an additional ultrafilter in reducing blood levels of several indicators of inflammation, ameliorating $\mathrm{Hb}$ concentration and reducing the level of ESA doses required.

It is significant that blood levels of CRP, SAA, C-5, MCP-1 and of several pro-inflammatory cytokines were lower at the end of the study phase in the present study. The reduction of markers of inflammation was likely promoted by the improved quality of dialysis-water obtained with the additional ultrafilter. Some evidence from the current literature may support this hypothesis despite the fact that levels of bacterial-derived DNA fragments were not assessed in our study. Biological tests adopt cytokine induction to detect the presence of bacterial fragments or bacterial products [9]; plasma endotoxin levels strongly correlate with serum CRP level [11]; high levels of short bacterial-derived-DNA fragments in dialysate increase CRP and interleukin (IL)-6 [6, $10,15-17]$; CPR is produced under the control of various pro-inflammatory cytokines, namely IL- 6 , IL- 1 , and TNF- $\alpha$ [1]; circulating bacterial-derived-DNA fragments are associated with higher levels of CRP and IL-6 [6].

Serum levels of SAA, C-5a and MCP-1 have never been taken into account in studies performed to assess the purity of dialysate. SAA is an important acute phase protein with different kinetics from CRP. It has been reported that SAA is a more sensitive indicator of inflammation in some noncardiovascular inflammatory conditions $[18,19]$. It plays a significant role in the atherosclerotic process; in association with other markers of inflammation, SAA contributes to early prediction of cardiovascular mortality $[18,19]$. C-5a is a protein fragment released from cleavage of complement component $\mathrm{C} 5$; it is a strong inflammatory peptide and a significant pathogenic driver of immuno-inflammatory diseases [20]. MCP-1 is a chemokine that controls the recruitment of leukocytes in inflammation and tissue injury as well as in bone marrow; this suggests that MCP-1 may play a role in hematopoiesis. In addition, MCP-1 may act as factor favoring lipid deposition in the arterial wall as well as being an accelerating factor of inflammation and atherosclerosis [21]. The role of this chemokine in inflammation-mediated diseases is supported by some evidence; in the absence of MCP-1 there was a substantial reduction of lipid deposition in the arterial wall [21]; increased plasma levels of MCP-1 following balloon angioplasty of coronary arteries predicted early restenosis, that may represent an accelerated form of atherosclerosis [21].

Beside the reduction of blood levels of pro-inflammatory cytokines, in our study we observed increased levels of antiinflammatory cytokines. The production of pro-inflammatory and anti-inflammatory cytokines is controlled by complex feedback mechanisms. Pro-inflammatory cytokines initiate defence against exogenous pathogens. However, overproduction of these mediators can be harmful. As a compensatory mechanism, anti-inflammatory cytokines down-regulate the exacerbated inflammatory process to maintain homoeostasis. The anti-inflammatory cytokines assessed in the present study belong to the class of cytokines activated by an acute or chronic phase of inflammation. For instance, IL-13 induces matrix metalloproteinases that protect against inflammation [22]; IL-1Ra is an acute phase protein important in host defense against endotoxin-induced injury by inhibiting the inflammatory responses caused by IL-1 [22]. We speculate that feedback or counter-regulatory mechanisms were active in our dialysis patients. Therefore, the reduction of inflammatory stimuli by improved dialysiswater triggered a compensatory reaction increasing antiinflammatory cytokines. Whether the anti-inflammatory response counteracting the pro-inflammatory cytokines may ameliorate outcomes remains to be assessed by other studies.

Interestingly, in the present study marked changes in blood levels of indicators of inflammation were observed while the bacterial count in water-dialysis remained stable after the additional ultrafilter. A plausible hypothesis is that the additional ultrafilter removed bacterial DNA fragments. In keeping with this hypothesis, it is reported that bacterial products or short bacterial DNA fragments have been found in dialysis-water regarded as bacteria-free on the basis of normal levels of endotoxin assessed by the LAL method $[6,7,23]$.

It is noteworthy that $\mathrm{Hb}$ levels increased at the end of the study phase (by 6\%) with lower ESA doses (23\% for epoietin alpha; $21 \%$ for darboepoietin, and 35\% for mircera) and ESA 
resistance index. We hypothesize that these changes were likely the result of improved inflammation and nutrition.

The trend in conventional indicators of nutrition [24] was not uniform in this study. Indeed, at the end of the study phase there were significant increases in mean blood level of triglycerides and albumin, but not in total cholesterol and serum phosphorus. The latter may explain the nonsignificant increase of mean parathormone (PTH) at the end of the study phase. All together, these data may indicate reduced inflammation as a plausible explanatory factor for the improved nutritional status. A more evident improvement of nutritional status has been reported by others as a positive effect of lower inflammation with ultrapure dialysate [11]. The shorter term of our observation period may explain the inconsistent results of the present study.

\section{Limitations and strengths}

Our study has several limitations. Bacterial fragments/products were not assessed. The study population was not large. The study was monocentric, and the length of observation was not long. Despite these limitations, the study has several strengths such as its cross-over design, and the simultaneous evaluation of pro- and anti-inflammatory cytokines, $\mathrm{Hb}$ levels and ESA doses changes.

\section{Conclusions}

The findings of the present study suggest that an additional ultrafilter may reduce activation of proinflammatory cytokines with beneficial effects on anemia. Therefore, further improvement of dialysis-water purity should be pursued to ameliorate the inflammatory status of patients on dialysis. However, these results should be regarded as hypothesis-generating only. Larger studies are mandatory to assess whether the routine use of an ultrafilter is associated to improved survival in dialysis patients.

\section{Compliance with ethical standards}

Conflict of interest The authors declare that they have no conflict of interest.

Ethical approval All procedures performed in this study were in accordance with the ethical standards of the institutional and/or national research committee and with the 1964 Helsinki declaration and its later amendments or comparable ethical standards.

Open Access This article is distributed under the terms of the Creative Commons Attribution 4.0 International License (http://creativecommons.org/licenses/by/4.0/), which permits unrestricted use, distribution, and reproduction in any medium, provided you give appropriate credit to the original author(s) and the source, provide a link to the Creative Commons license, and indicate if changes were made.

\section{References}

1. Arici M, Walls J (2001) End stage renal disease, atherosclerosis, and cardionascular mortality. Kidney Int 59:407-414

2. Di Lullo L, House A, Gorini A et al (2015) Chronic kidney disease and cardiovascular complications. Heart Fail Rev 20:259-272

3. Morrone LF, Mazzaferro S, Russo D et al (2009) Interaction between parathyroid hormone and the Charlson comorbidity index on survival of incident haemodialysis patients. Nephrol Dial Transpl 24:2859-2865

4. De Francisco LMA, Stenvinkel P, Vaulant S (2009) Inflammation and its impact on anemia in chronic kidney disease: from hemoglobin variability to hyporesponsiveness. Nephrol Dial Transpl $2: 18-26$

5. Goichoecea M, Martin J, De Seguera P et al (1998) Role of cytokines in the response to erythropoetin in hemodialysis patients. Kidney Int 54:1337-1343

6. Bossola M, Sanguinetti M, Scribano D et al (2009) Circulating bacterial-derived DNA fragments and markers of inflammation in chronic hemodialysis patients. Clin J Am Soc Nephrol 4:379-385

7. Gomila M, Gasco J, Gil J et al (2006) A molecular microbial ecology approach to studying hemodialysis water and fluid. Kidney Int 70:1567-1576

8. Penne EL, Visser L, van den Dorpel MA et al (2009) Microbiological quality and quality control of purified water and ultrapure dialysis fluids for online hemodiafiltration in routine clinical practice. Kidney Int 76:665-672

9. Schindler R, Beck W, Deppisch R et al (2004) Short bacterial DNA fragments: detection in dialysate and induction of cytokines. J Am Soc Nephrol 15:3207-3214

10. Cazzavillan S, Ratanarat R, Segala C et al (2007) Inflammation and subclinical infection in chronic kidney disease: a molecular approach. Blood Purif 25:69-76

11. Ching-Ha Kwan B, Chow KM et al (2013) Effect of using ultrapure dialysate for hemodialysis on the level of circulating bacterial fragment in renal failure patients. Nephron Clin Pract 123:246-253

12. Russo D, Morrone LF, Brancaccio S et al (2009) Pulse pressure and presence of coronary artery calcification. Clin J Am Soc Nephrol 4:316-322

13. Di Iorio B, Nargi O, Cucciniello E et al (2011) Coronary artery calcification progression is associated with arterial stiffness and cardiac repolarization deterioration in hemodialysis patients. Kidney Blood Press Res 34:180-187

14. Russo D, Battaglia Y, Buonanno E (2010) Phosphorus and coronary calcification in predialysis patients. Kidney Int 78:818

15. Szeto CC, Kwan BCH et al (2015) Circulating bacterial-derived DNA fragment level is a strong predictor of cardiovascular disease in peritoneal dialysis patients. PLOS One. doi:10.1371/journal. pone. 0125162

16. Perri A, Vizza A, Lupinacci S et al (2016) Adiponectin secreted by tubular renal cells during LPS exposure worsens the cellular inflammatory damage. J Nephrol 29:185-194

17. Tessitore N, Poli A, Bedogna V et al. (2017) A single dialysis session of hemodiafiltration with sorbentregenerated endogenous ultrafiltrate reinfusion (HFR) removes hepcidin more efficiently than bicarbonate hemodialysis: a new approach to containing hepcidin burden in dialysis patients? J Nephrol. doi:10.1007/s40620017-0383-0 (Epub ahead of print)

18. Malle E, Steinmetz A, Raynes JG (1993) Serum amyloid A (SAA): an acute phase protein and apolipoprotein. Atherosclerosis 102:131-146

19. Morrow DA, Rifai N, Antman EM et al (2000) Serum amyloid A predicts early mortality in acute coronary syndromes. J Am Coll Cardiol 35:358-362 
20. Minty A, Chalon P, Derocq JM et al (1993) Interleukin-13 is a new human lymphokine regulating inflammatory and immune responses. Nature 362:248-250

21. Deshmane SL, Kremlev S, Amini S et al (2009) Chemoattractant protein-1 (MCP-1): an overview. J Interferon Cytokine Res 6:313-326

22. Arend W, Malyak M, Guthridge CJ et al (1998) Interleukin-1 receptor antagonist: role in biology. Annu Rev Immunol 16:27-55
23. Navarro MD, Carracedo J, Ramirez R et al (2007) Bacterial DNA prolongs the serviva of inflammed mononuclear cells in hemodialysis patients. Nephrol Dial Transpl 22:3580-3585

24. Marcelli D, Wabel P, Wieskotten s et al (2015) Physical methods for evaluating the nutrition status of hemodialysis patients. $\mathbf{J}$ Nephrol 28:523-530 